TITLE:

\title{
Phase separation and dewetting in polystyrene/poly(vinyl methyl ether) blend thin films in a wide thickness range
}

\section{AUTHOR(S):}

OGAWA, Hiroki; KANAYA, Toshiji; NISHIDA, Koji; MATSUBA, Go

\section{CITATION:}

OGAWA, Hiroki ...[et al]. Phase separation and dewetting in polystyrene/poly(vinyl methyl ether) blend thin films in a wide thickness range. Polymer 2008, 49(1): 254-262

\section{ISSUE DATE:}

2008-01-10

URL:

http://hdl.handle.net/2433/93443

\section{RIGHT:}

Copyright @ 2007 Elsevier; この論文は出版社版でありません。引用の 際には出版社版をご確認ご利用ください。; This is not the published version. Please cite only the published version. 


\title{
Phase Separation and Dewetting in Polystyrene/Poly(vinyl methyl ether) Blend Thin Films in a Wide Thickness Range
}

\author{
Hiroki Ogawa, Toshiji Kanaya*, Koji Nishida and Go Matsuba \\ Institute for Chemical Research, Kyoto University, Kyoto, Japan
}

$\begin{array}{ll}\text { corresponding author: } & \text { T. Kanaya } \\ \text { Postal address: } & \text { Institute for Chemical Research, Kyoto University } \\ & \text { Uji, Kyoto-fu, 611-0011 } \\ \text { Tel. \& Fax.: } & +81-774-38-3140,+81-774-38-3146 \\ \text { email: } & \text { kanaya@scl.kyoto-u.ac.jp }\end{array}$




\section{Abstract}

Phase separation and dewetting processes of blend thin films of polystyrene (PS) and poly(vinyl methyl ether) (PVME) in two phase region have been studied in a wide film thickness range from $65 \mu \mathrm{m}$ to $42 \mathrm{~nm}\left(\sim 2.5 R_{\mathrm{g}}, R_{\mathrm{g}}\right.$ being radius of gyration of a polymer $)$ using optical microscope (OM), atomic force microscope (AFM) and small-angle light scattering (LS). It was found that both of the phase separation and dewetting processes depend on the film thickness, and were classified into four thickness regions. In the first region above $\sim 15 \mu \mathrm{m}$ the spinodal decomposition (SD) type phase separation occurs in a similar manner to bulk and no dewetting is observed. This region can be regarded as bulk. In the second region between $\sim 15$ and $\sim 1 \mu \mathrm{m}$, the SD type phase separation proceeds in the early stage while the characteristic wavelength of the SD decreases with the film thickness. In the late stage dewetting is induced by the phase separation. In the third region between $\sim 1 \mu \mathrm{m}$ and $\sim 200 \mathrm{~nm}$ the dewetting is observed even in the early stage. The dewetting morphology is very irregular and no definite characteristic wavelength is observed. It is expected that the irregular morphology is induced by mixing up the characteristic wavelengths of the phase separation and the dewetting. In the forth region below $\sim 200 \mathrm{~nm}$ the dewetting occurs after a long incubation time with a characteristic wavelength, which decreases with the film thickness. It is considered that the layered structure is formed in the thin film during the incubation period and triggers the dewetting through the capillary fluctuations mechanism or the composition fluctuations one. 


\section{Introduction}

Properties of polymer thin films on solid substrates are of great importance from scientific as well as industrial viewpoints because many important phenomena are related to the surface properties such as coating, adhesive, surface friction, lubricants, and dielectric layer [1-4]. Many studies on single component thin films have been carried out to reveal interesting but unusual properties of thin films. It was reported by many groups [5-9] that the glass transition temperature $T_{\mathrm{g}}$ decreased with film thickness below about $40 \mathrm{~nm}$, and the thermal expansivity of the thin films is also a big issue under debate in the thin film research field [8-13]. Recently, dewetting and phase separation of polymer blend thin films are also of great interest because of technological applications as well as fundamental investigations. The dewetting of the phase separated polymer blend thin film limits the industrial applications because they require a stable, homogeneous and uniform thin films. This motivated the fundamental studies of polymer blend thin films very much to reveal the dewetting mechanism and control the morphology [4]. Stamm et al. [14] have extensively investigated blend thin films of poly ( $p$-methylstyrene) (PpMS) and deuterated polystyrene (dPS), which was a weakly incompatible system, to find that bilayer structure is formed via phase separation and the upper layer of poly( $p$-methylstyrene) dewetted on the lower layer of deuterated polystyrene. The blend thin films of poly (styrene-ran-acrylonitrile) (SAN) and deuterated poly (methyl methacrylate) (dPMMA) were also studied by some groups [1520]. Composto et al. recently examined the kinetics of phase separation in the blend thin films displaying discrete and bicontinuous domain morphologies [15], and found breakdown of dynamic scaling in the bicontinuous domain growth due to suppression of lateral hydrodynamic pumping with decreasing the film thickness while the scaling was 
hold in the discrete case. The same group also studied the dewetting mechanism of thin blend films of SAN and DPMMA in a wide thickness range [16-18] and presented a morphology map [19] based on the pattern development mechanism for thickness values and bulk composition between $50-1000 \mathrm{~nm}$ and $\phi_{\mathrm{PMMA}}=0.3-0.8$, respectively. Recent works by Liao et al. [20] have experimentally demonstrated the dewetting mechanism induced by composition fluctuations [21-24] for thermodynamically stable ultrathin blend films of SAN and DPMMA in one phase region. In the two phase region dewetting of the whole film is followed by the phase separation in the droplets.

As for thin films of polystyrene (PS) and poly (vinyl methyl ether) (PVME) blend, which has a lower critical solution temperature (LCST) type phase diagram in bulk, Tanaka et al. [25] studied the surface structure of PS/PVME ultrathin films using atomic force microscopy (AFM) and observed dewetted droplets in one phase region for films thinner than twice the radius of gyration. Karim et al. [26, 27] also reported similar undulation of the free surface in the two phase region where dPS is encapsulated by PVME in the thin film. These observations suggest that phase separation and dewetting competitively occur in ultrathin films of polymer blend. Small-angle light scattering studies on the kinetic of phase separation of PS/PVME blend thin films [28] show that the phase separation proceeds by a spinodal decomposition mechanism in a thick film $(=180 \mathrm{~nm})$ while in the thin film $(=30 \mathrm{~nm})$ it switches to nucleation and growth. However, there are no systematic studies on thin blend films of PS and PVME in a wide film thickness range.

In this study, therefore, we investigated morphology and kinetics of phase separation as well as dewetting in thin films of PS/PVME blends as a function of film thickness using light scattering (LS) techniques and optical microscope (OM) as well as 
using atomic force microscopy (AFM) in a wide thickness $d$ range from semi-infinite thickness $(65 \mu \mathrm{m})$ to that close to radius of gyration $R_{\mathrm{g}}$ of PS and PVME chains $(d \sim$ $2.5 R_{\mathrm{g}}$ ) to elucidate the relation between phase separation and dwetting in blend thin films of PS and PVME in the two phase region.

\section{Experiment}

Polystyrene (PS) and poly(vinyl methyl ether) (PVME) used in this study have weight -average molecular weights $M_{\mathrm{w}}=280,000$ and 90,000, and the molecular weight distributions in terms of $M_{\mathrm{w}} / M_{\mathrm{n}}=3.01$ and 1.88, respectively, where $M_{\mathrm{n}}$ is number average molecular weight. Both of PS and PVME were purchased from Scientific Polymer Products, Inc. PS and PVME were purified by precipitating the toluene solutions into excess methanol and $n$-heptane several times, respectively, and dried in vacuum at room temperature for $72 \mathrm{~h}$. It is noted that PS and PVME used in this experiment have similar values of radius of gyration $R_{g}$, which are $16.6 \mathrm{~nm}$ and $16.2 \mathrm{~nm}$, respectively, although their molecular weights are different. Films below and above 1 $\mu \mathrm{m}$ were prepared by spin-coating and casting the toluene solution of PS and PVME, respectively, on a cleaned glass substrate after filtering with $2 \mu \mathrm{m}$ pore size membrane at room temperature. The films were then annealed at $60{ }^{\circ} \mathrm{C}$ for $24 \mathrm{~h}$ after drying in vacuum at room temperature for $24 \mathrm{~h}$. Thickness of polymer film was controlled by varying the polymer concentration in solution and confirmed with ellipsometer measurements.

Small-angle light scattering (LS) measurements were carried out using home-made apparatus with confocal collimation, which enables us to access a very low $q$ range down to $0.1 \mu \mathrm{m}^{-1}$ with extremely low background. These characteristic features unable 
us to measure such thin film as $42 \mathrm{~nm}$. Two dimensional scattering intensities were accumulated every $5 \mathrm{~min}$ after temperature jump from one phase region to two phase region $\left(=115^{\circ} \mathrm{C}\right)$. Details of the LS instrument were reported elsewhere [29]. Optical microscope $(\mathrm{OM})$ measurements were also done after temperature jump from one phase region to two phase region $\left(=115^{\circ} \mathrm{C}\right)$ using OLYMPUS BX50 equipped with a CCD camera. Atomic force microscope (AFM) measurements were performed at room temperature after quenching the sample from two phase region $\left(=115^{\circ} \mathrm{C}\right)$ using JEOL JSPM-4200 to examine the surface morphology of the films.

\section{Results and Discussion}

First of all we have constructed a phase diagram of the bulk PS/PVME blend. We observed LS intensity from samples with various blend ratios during the heating process from room temperature with a rate of $1{ }^{\circ} \mathrm{C} / \mathrm{min}$. It was reported [30] that the phase diagram dose not depend on the heating rate when it is below $2{ }^{\circ} \mathrm{C} / \mathrm{min}$. In this measurement the cloud point was defined as an onset temperature of the scattering intensity. The phase diagram thus obtained is shown in Figure 1, which is a lower critical solution temperature (LCST) type. The estimated critical weight fraction of PS $\phi_{\mathrm{PS}}$ is 0.3 and the critical temperature $T_{\mathrm{c}}$ is $104.6{ }^{\circ} \mathrm{C}$. At $115{ }^{\circ} \mathrm{C}$ the coexisting composition are nearly pure components, which were estimated by extrapolating the phase diagram in Figure 1. The glass transition temperature $T_{\mathrm{g}}$ of the bulk blend with the critical concentration $\left(\phi_{\mathrm{PS}}=0.3\right)$ is $-20{ }^{\circ} \mathrm{C}$. The phase diagram is almost identical to the reported one [31]. In this work all LS, OM and AFM measurements were performed on PS/PVME blend thin films with the critical concentration of $\phi_{\mathrm{PS}}=0.3$. 
OM measurements were performed on the blend thin films as a function of annealing time after temperature jump to $115^{\circ} \mathrm{C}$ in two phase region from one phase region. In addition the surface morphology was examined by AFM with contact mode after quenching the film to room temperature after $90 \mathrm{~min}$ annealing at $115{ }^{\circ} \mathrm{C}$. The OM images are shown in Figure 2 for $65 \mu \mathrm{m}, 9 \mu \mathrm{m}, 2 \mu \mathrm{m}, 466 \mathrm{~nm}, 98 \mathrm{~nm}$ and $42 \mathrm{~nm}$ films at 20, 50, 90 and 120 min after the temperature jump, and the AFM results are given in Figure 3. In the following we will discuss the OM and AFM results in order of the film thickness.

In the case of $65 \mu \mathrm{m}$ film, the observed OM patterns look like typical SD phase separation process although they are $2 \mathrm{D}$ images, and the AFM results show the surface is very smooth. These observations suggest that the SD type phase separation proceeds inside the $65 \mu \mathrm{m}$ film. For the 9 and $2 \mu \mathrm{m}$ films similar SD patterns are observed at 20 min in the early stage although the characteristic wavelengths seem smaller than that in the $65 \mu \mathrm{m}$ film. The surfaces of the 9 and $2 \mu \mathrm{m}$ films are very smooth, at least at 20 min, which was confirmed by AFM measurements. As the phase separation proceeds some semi-irregular spot-like patterns with strong (white) contrast are observed over the SD pattern at 90 and $120 \mathrm{~min}$ in the later stage. This is well recognized in the OM image at $120 \mathrm{~min}$ for the $2 \mu \mathrm{m}$ film (Figure 2(c)). The AFM result in Figure 3(b) clearly shows that the white spots are holes on the surface, confirming that the semiirregular pattern is due to dewetting.

In the $466 \mathrm{~nm}$ film, the observed OM images are so irregular even at 20 and $50 \mathrm{~min}$ and grow with annealing time keeping the irregularity as seen in Figure 2(d). The surface morphology examined by AFM confirmed that the irregular pattern was due to irregular dewetting morphology. As the film thickness further decreases, the time 
evolution of the OM and AFM images very much changed for the 98 and $42 \mathrm{~nm}$ films. In the first 20 or $30 \mathrm{~min}$ the $\mathrm{OM}$ images are very homogeneous and the surfaces examined by AFM are very smooth, implying nothing happens. After the long incubation period regular bicontinuous morphologies with strong contrast are observed in the $\mathrm{OM}$ images, and finally become isolated droplets at $120 \mathrm{~min}$. The AFM images of the $98 \mathrm{~nm}$ after $90 \mathrm{~min}$ annealing at $115^{\circ} \mathrm{C}$ in Figures 3(d) clearly shows that the regular pattern is due to the dewetting. The present OM and AFM results show that the phase separation as well as dewetting in the PS/PVME blend thin film depend on the film thickness very much.

Concerning the dewetting in the blend thin films, it is emphasized that dewetting in one component (PS or PVME) films dose not occur in such thick films as examined here. For example, dewetting occurs in PS films below about 5-6 nm [32] while it depends on temperature and molecular weight more or less. In any case, such thick one component films as $470 \mathrm{~nm}$ do not show dewetting. We also studied the stability of the blend films in a range from $42 \mathrm{~nm}$ to $65 \mu \mathrm{m}$ at $90{ }^{\circ} \mathrm{C}$ in one phase region and found that no dewetting was observed during annealing at $90{ }^{\circ} \mathrm{C}$ for $6 \mathrm{~h}$ even in the 42 $\mathrm{nm}$ film, showing that the blend films are stable in one phase region. The present results suggest that dewetting observed in the PS/PVME thin films is induced by the phase separation.

In the next step, we studied the phase separation and dewetting of the PS/PVME blend thin films using time-resolved LS just after temperature jump to $115^{\circ} \mathrm{C}$ in two phase region from one phase region. Figures 4(a)-(f) show the time evolutions of one dimensional (1D) LS profiles for the $65 \mu \mathrm{m}, 9 \mu \mathrm{m}, 2 \mu \mathrm{m}, 466 \mathrm{~nm}, 98 \mathrm{~nm}$ and $42 \mathrm{~nm}$ films, which were obtained by circular averaging the $2 \mathrm{D}$ scattering profiles. The 
scattering profiles and their time evolutions clearly depend on the film thickness as observed in the OM and AFM measurements. In the $65 \mu \mathrm{m}$ film the scattering profile shows a peak and its position gradually shifts to lower $q$ with annealing time, showing typical spinodal decomposition (SD) type phase separation kinetics. In a rather thin film such as $2 \mu \mathrm{m}$, however, the time evolution of the scattering profile is different. In an early stage before $\sim 30$ min the time evolution of the scattering profile is very similar to that in the $65 \mu \mathrm{m}$ film, however, the growth in the peak intensity is slowed down and the intensity in a low $q$ range below the peak grows in a late stage after $\sim 60 \mathrm{~min}$. This must be caused by the semi-irregular holes on the surface due to the dewetting as seen in OM images (see Figures 2(b) and (c)).

As the film thickness further decreases, the scattering peak disappears for films below $\sim 1 \mu \mathrm{m}$ as seen for the $466 \mathrm{~nm}$ film in Figure 4(d). This must correspond to the irregular dewetting morphology observed in the OM and AFM measurements (see Figure 2(d) and Figure 3(c)). The scattering pattern obtained by fast Fourier transform (FFT) of the OM image of the $466 \mathrm{~nm}$ film does not show any peaks in the low $q$ range down to $0.04 \mu \mathrm{m}^{-1}$, confirming that there are not characteristic wavelengths in the spatial scale below $\sim 150 \mu \mathrm{m}$. With further decreasing the film thickness, surprisingly, the peak appears again below $\sim 200 \mathrm{~nm}$ and the incubation time before appearing the peak is very long.

For quantitative discussions we plotted the peak intensity $I_{\max }$ and the peak position $q_{\text {max }}$ as a function of annealing time in Figures 5(a) and (b), respectively, for the $65 \mu \mathrm{m}$, $14 \mu \mathrm{m}, 9 \mu \mathrm{m}, 2 \mu \mathrm{m}, 98 \mathrm{~nm}$ and $42 \mathrm{~nm}$ films. We first examine the results of the 65 $\mu \mathrm{m}$ film. The scattering peak appears at around $2 \mu \mathrm{m}^{-1}$ at $\sim 7 \mathrm{~min}$ after the temperature 
jump and the scattering intensity exponentially increases with annealing time before $\sim 7$ min. After $\sim 7$ min, as can be seen in Figures 5(a) and (b), the peak intensity $I_{\max }$ and the peak position $q_{\max }$ increase according to power laws $I_{\max }(t) \sim t^{\beta}$ and $q_{\max }(t) \sim t^{-\alpha}$, respectively, suggesting that the scaling relations are hold. At around 30 min the slopes in $q_{\max }(t) \sim t^{-\alpha}$ and $I_{\max }(t) \sim t^{\beta}$ become steeper. The exponents $\alpha$ and $\beta$ evaluated from the observed time evolutions of $I_{\max }$ and $q_{\max }$ are $\alpha=0.25$ and $\beta=0.85$ before 30 $\min$ and $\alpha=0.54$ and $\beta=1.62$ after $30 \mathrm{~min}$, respectively, for the $65 \mu \mathrm{m}$ film. These values are very close to those reported for the PS/PVME bulk blend by Hashimoto et al. $[33,34]$, and the relations $\beta>3 \alpha$ and $\beta=3 \alpha$ are hold before and after $30 \mathrm{~min}$, respectively. According to Hashimoto et al. the former and the latter can be regarded as the intermediate stage and the late stage of spinodal decomposition (SD) type phase separation, respectively [34]. From these LS results, we concluded that the $65 \mu \mathrm{m}$ film could be regarded as a bulk sample. As mentioned above, we did not observe a scattering peak in the very early stage before $\sim 7 \mathrm{~min}$. This was also observed in the experiments by Hashimoto et al. [33, 34].

The phase separation kinetics is almost independent of the film thickness for the films above $\sim 15 \mu \mathrm{m}$. This region is termed Region I hereafter. On the other hand, as the film thickness decreases below $\sim 15 \mu \mathrm{m}$, effects of the film thickness gradually appear on the kinetics. In the case of $14 \mu \mathrm{m}$ film, the phase separation kinetics seems to be identical with that in the $65 \mu \mathrm{m}$ film (bulk), at least, until $\sim 20 \mathrm{~min}$ after the temperature jump as seen in Figure 5. However, they deviate from the bulk behavior at $\sim 20$ min, showing some effects of film thickness. For films below $\sim 15 \mu \mathrm{m}$ and above 
$\sim 1 \mu \mathrm{m}$, we observed similar level-off of the peak intensity after $\sim 20 \mathrm{~min}$. This region is termed Region II.

In order to see the differences between Region I and Region II, we plotted the characteristic wavelength $\lambda_{\max }\left(=2 \pi / q_{\max }\right)$ at $10 \mathrm{~min}$ after the temperature jump as a function of the film thickness in Figure 6. In the figure the characteristic wavelength for the films below $200 \mathrm{~nm}$ is also plotted, which will be discussed later. The characteristic wavelength $\lambda_{\max }$ is almost constant for the films above $\sim 15 \mu \mathrm{m}$ (Region I), however, it begins to decrease with film thickness between $\sim 15$ and $\sim 1 \mu \mathrm{m}$ (Region II). When the characteristic wavelength $\lambda_{\max }$ encounters the film thickness $d$ (thick solid line in Figure 6) the characteristic peak disappears in the scattering profile, suggesting cut-off of the fluctuations with longer wavelength than the film thickness. In Figure 7, the LS profiles at $10 \mathrm{~min}$ in the early stage after temperature jump are shown for the 2, 4 and $65 \mu \mathrm{m}$ (bulk) films, the intensities of which were normalized to that at $q=5 \mu \mathrm{m}^{-1}$ to compare the shapes of profiles. In the figure, $q$ values corresponding to the film thickness $(q=2 \pi / d)$ are indicated by down arrows. It is very clear that the scattering intensity in a $q$ range lower than the scattering vector corresponding to the film thickness is suppressed, meaning that the fluctuations with wavelength longer than the film thickness are hard to appear in the thin films.

As the film thickness further decreases below $\sim 1 \mu \mathrm{m}$, the scattering profiles are very different from those above $\sim 1 \mu \mathrm{m}$. As demonstrated for the $466 \mathrm{~nm}$ film (see Figure 4(d)), no scattering peak is observed during the time evolution in the film thickness range between $\sim 1 \mu \mathrm{m}$ and $\sim 200 \mathrm{~nm}$, which is termed Region III. As shown in the OM and AFM measurements (Figure 2(d) and Figure 3(c)) this is due to the 
irregular dewetting morphology. However, at the moment we have no final conclusions on the irregular pattern. One possibility is that the phase separation and dwetting occur simultaneously and their characteristic wavelengths are mixed up, resulting in the irregular morphology.

For the films below $200 \mathrm{~nm}$ (Region IV) a scattering peak appears again (see Figure 2). The time evolutions of the peak intensity $I_{\max }$ and the peak position $q_{\max }$ are shown for the $98 \mathrm{~nm}$ and $42 \mathrm{~nm}$ films in Figures 5(a) and (b). This characteristic wavelength $\lambda_{\max }\left(=2 \pi / q_{\max }\right)$ is due to the dewetting as confirmed in the AFM measurements. The characteristic wavelength of dewetting in the early stage for the 98 $\mathrm{nm}$ film is larger than that of the SD phase separation in the films above $\sim 15 \mu \mathrm{m}$ (Figure 6), and decreases with the film thickness. This is one of the characteristic features of dewetting in the PS/PVME thin films below $\sim 200 \mathrm{~nm}$ (Region IV). Another characteristic feature is a very long incubation time before appearance of the scattering peak, which are $\sim 30 \mathrm{~min}$ and $\sim 40 \mathrm{~min}$ for the $98 \mathrm{~nm}$ and $42 \mathrm{~nm}$ films, respectively. During the incubation period the scattering intensities hardly grow, while they increase very rapidly after the incubation period (see Figure 5(b)) according to a power law with unexpected large exponents. In the following we will discuss the dewetting mechanisms in Region II and IV.

In the region II, for example, we consider the $2 \mu \mathrm{m}$ film. The scattering peak was observed at around $q_{\max }=5 \mu \mathrm{m}^{-1}$ in the early stage of phase separation and gradually shifted to lower $q$ with annealing time (Figure 5(b)). When $q_{\max }$ comes close to the film thickness $\left(q=2 \pi / 2 \sim 3 \mu \mathrm{m}^{-1}\right)$ at $\sim 25 \mathrm{~min}$, the growth of scattering intensity slows down or stops. Similar tendency was observed in the 9 and $14 \mu \mathrm{m}$ films in Region II. From the OM results, it was also observed that the growth of phase separation stopped 
and the dewetting began to occur. These observations show that the composition fluctuations due to the SD phase separation induce the in-plane fluctuations on the surface/interface and rupture the film. An important problem we have to clarify is how the SD composition fluctuations induce the dewetting. Recently Chung et al. [35] have shown that phase separation drove dewetting in blend films of poly(methyl methacrylate) (PMMA) and poly(styrene-ran-acrylonitrile) (SAN) $(d=550 \mathrm{~nm})$, and also shown that the capillary fluctuations failed to explain the roughening (or dewetting). On the basis of a universal scaling between the long wavelength fluctuations and surface roughness, they proposed that spinodal decomposition during initial phase separation may trigger the long wavelength fluctuations, and pointed out a possibility that the long wavelength fluctuations were created through the composition fluctuation mechanism proposed by Clarke [24]. In Region II, thus the dewetting induced by the SD composition fluctuations is one possible mechanism.

In Region IV, the regular patterns in the dewetting process suggest that the dewetting process proceeds through the SD mechanism. Generally speaking, however, SD type dewetting occurs when the system is in an unstable region and no incubation period is required $[36,37]$. The long incubation time means that some structure is formed before the dewetting in the thin film and the dewetting may be triggered by the structure. In the incubation period we see the very small increase in the LS intensity (see Figure 5(a)) although it is not the SD type phase separation observed in Region II. This may suggest that some composition fluctuations proceed along the normal direction to the surface to form some layer structure, which are hard to be seen in the present scattering geometry with incident beam normal to the film surface. Such layer structure was reported for other polymer blend thin films. Stamm et al. [14] studied 
dewetting and phase separation in thin films of a weakly imcompatible polymer blend of deuterated polystyrene (dPS) and poly(p-methylstyrene) (PpMS), and found that the interactions with substrate during the phase separation leaded to a bi-layer formation with PpMS on the top layer. The bi-layer structure is unstable and defines the starting point for the dewetting on the top of the dPS layer. In the final dewetting state a homogeneous layer of dPS on the top of the substrate is covered with ultra-thin PpMS layer as well as the quite thick mesoscopic drops of PpMS. Apparently this mechanism is similar to our case. However, we observed bear Si surface in the AFM measurements, and hence no homogeneous layer of PVME was suggested. Blend thin films of deuterated poly(methyl methacrylate) (dPMMA) and poly(styrene-ranacrylonitrile) (SAN) were also extensively studied by Composte et al. [15-18] in a wide thickness $d$ range from semi-infinite limit to less than radius of gyration $R_{\mathrm{g}}$ of a polymer chain. In the semi-infinite limit they observed the so-called surface directed spinodal decomposition (SDSD), which is suppressed when the characteristic length is below the film thickness. In the thin film regions of $10 R_{\mathrm{g}}<d<150 R_{\mathrm{g}}$ and of $\sim R_{\mathrm{g}}<d<10 R_{\mathrm{g}}$, tri-layer structure is formed in the early stage, consisting of surface and interface dPMMA-rich layers and middle SAN-rich layer. In the SAN-rich middle layer they observed phase separated structure and homogeneous structure for the former and the latter, respectively. These reported results tell us that layered structure is easily formed due to the interactions between one of blend component polymer and substrate (and/or air), and triggered the dewetting. We therefore expect tri-layer structure formation due to the preferential interactions between PVME and substrate at the interface and between PVME and air at the surface [38] before dewetting and the preliminary result of neutron reflectivity (NR) measurements on the deuterated PS/PVME blend thin films 
supported the tri-layer structure [39]. Note that the surface and interface segregation of PVME reduces the concentration of PVME in the middle bulk-like layer, but the preliminary NR results suggest that it is negligible for the films above $\sim 50 \mathrm{~nm}$ [39].

For dewetting, the thin film must be ruptured by some mechanism. It was reported for dPMMA/SAN blend thin films $\left(10 R_{\mathrm{g}}<d<150 R_{\mathrm{g}}\right)[18]$ that the lateral phase coarsening occurs in the middle stage after the tri-layer structure formation, and finally the capillary fluctuations causes the thickness to vary and rapture the middle layer. Assuming the tri-layer structure formation in the PS/PVME blend thin film below $\sim 200 \mathrm{~nm}\left(\sim 13 R_{\mathrm{g}}\right)$ before dewetting, the situation is very similar to the dPMMA/SAN blend thin film. According to Composto et al. [17] the characteristic wavelength of the capillary fluctuations $\Lambda_{m}$ is given by

$$
\Lambda_{m}=h_{0}^{2}\left[4 \pi\left(\gamma_{1}+\gamma_{12}\right) / A\right]^{1 / 2}
$$

for tri-layer structure. Here, $h_{0}$ is the initial thickness of the middle layer, $\gamma_{1}$ and $\gamma_{12}$ are the surface and interfacial tensions of the top layer, and $A$ is the Hamaker constant. Eq. (1) predicts that the characteristic wavelength of the dewetting decreases with the film thickness. This agrees with our observation shown in Figure 5 although the detailed comparison is not impossible at the moment.

Recently An and co-workers [20] have explained the dewetting of thermodynamically stable blend ultrathin film of PMMA and SAN $\left(d \sim R_{\mathrm{g}}\right)$ in terms of a dewetting mechanism induced by composition fluctuations [20-23], which is a similar mechanism proposed in Region II This mechanism may be possible even in two phase region. PVME has attractive interactions with Si substrate and air [38], and hence 
would diffuse to the substrate so as to create the composition gradient across the film. The diffusion could not occur homogeneously over the film surface to create the composition fluctuations in the mixture along the surface. When the amplitude of the fluctuations is large enough, the free surface is eventually destabilized, leading to the dewetting. At the moment we have no direct evidence for the dewetting mechanism in Region IV, but cannot deny both of them as a rapture mechanism for blend thin film. Investigation in the composition fluctuations normal to the surface would be give us significant information on the dewetting mechanism.

\section{Conclusion}

In this study we investigated the morphology and the kinetics of phase separation and dewetting in blend thin films of PS/PVME as a function of film thickness from 42 $\mathrm{nm}$ to $65 \mu \mathrm{m}$ using OM, AFM and LS. The time evolutions of OM images and LS patters after the temperature jump into the two phase region strongly depend on the film thickness, and could be classified into four regions (Regions I, II, III and IV). In Region I above $\sim 15 \mu \mathrm{m}$, the phase separation kinetics can be explained as normal SD phase separation in bulk. As the film thickness decreases, the characteristic wavelength to the SD phase separation becomes smaller with the film thickness in Region II between $\sim 15$ and $\sim 1 \mu \mathrm{m}$ because the composition fluctuations are suppressed by the film thickness. In Region II the films finally dewet in a very late stage, depending on the film thickness, which must be induced by the phase separation. In Region IV below $\sim 200 \mathrm{~nm}$ the films dewet after a long incubation time. The bicontinuous morphologies with characteristic wavelength $\lambda_{\max }$ implies that the dewetting mechanism is a SD type, and the long incubation time was assigned to tri- 
layer structure formation. In Region III $(1 \mu \mathrm{m} \sim 200 \mathrm{~nm})$ between Region II and Regio IV the LS patterns do not show any characteristic scattering peaks, corresponding to the irregular dewetting pattern confirmed by AFM. It suggests that the phase separation and dewetting occur simultaneously and their characteristic wavelengths are mixed up, resulting in the irregular morphology. In Regions II and IV the dewetting mechanisms were discussed. In Region II the dewetting induced by the SD composition fluctuations was proposed. In Region IV below $200 \mathrm{~nm}$ preferential interactions between one component of blend polymers (PVME) and substrate as well as air lead to vertical phase separation to create tri-layer structure, consisting of interface and surface PVME layers and the middle blend layer. For dewetting, the middle layer must be ruptured by some mechanism. One possibility is the capillary fluctuations and the other is the composition fluctuations at the interlayer. This will be confirmed in near future by neutron reflectivity measurements.

\section{References}

[1] Jones R L, Richards R W. Polymers at Surface and Interfaces. Cambridge: Cambridge University Press; 1999.

[2] Karim A, Kumar S. Polymer Surfaces, Interfaces and Thin Films. Singapore: World Scientific; 2000.

[3] Bucknall D G. Progress in Materials Science 2004; 49: 713-786.

[4] Mueller-Buschbaum P, Bauer E, Wunnicke O, Stamm M. J. Phys.: Condens. Matter 2005; 17: S363-S386. 
[5] Keddie J L, Jones R A, Cory R A. Europhys. Lett. 1994; 27: 59-64.

[6] Keddie J L, Jones R A, Cory R A. Faraday Discuss. 1994; 98: 219-230.

[7] Forrest J A, Dalnoki-Veress K, Stevens J R, Dutcher J R. Phys. Rev. Lett. 1996; 77: 2002-2005.

[8] Kawana S, Jones R A L. Phys. Rev. E 2001; 63: 021501-1-021501-6.

[9] Miyazaki T, Nishida K, Kanaya T. Phys. Rev. E 2004; 69: 06183-06188.

[10] Orts W J, Zanten J H v, Wu W, Satija S K. Phys. Rev. Lett. 1993; 71: 867-870.

[11] Fukao K, Miyamoto Y. Phys. Rev. E 2000; 61: 1743-1754.

[12] DeMaggio G B, Frieze W E, Gidley D W, Zhu M, Hristov H A, Yee A F. Phys. Rev. Lett. 1997; 78: 1524-1527.

[13] Kanaya T, Miyazaki T, Watanabe H, Nishida K, Yamano H, Tasaki S, Bucknall D B. Polymer 2003; 44: 3769-3773.

[14] Mueller-Buschbaum P, O'Neil S A, Affrossman S, Stamm M. Macromolecules 1998; 31: 5003-5009.

[15] Chung H, Composto R J. PHys. Rev. Lett. 2004; 92: 185704-185707.

[16] Wang H, Composto R J. Europhys. Lett. 2000; 50: 622-627.

[17] Wang H, Composto R J. J. Chem. Phys. 2000; 113: 10386-10397.

[18] Wang H, Composto R J. Interface Science 2003; 11: 237-248.

[19] Chung H -j, Wang H, Composto R J. Macromolecules, 2006; 39: 153-161. 
[20] Liao Y, Su Z, Sun Z, Shi T, An L. Macromol. Rapid Commun. 2006; 27: 351355.

[21] Wensink K D F, Je'remoe B. Langmuir 2002; 18: 413-416.

[22] Sharma A, Mittal J. Phys. Rev. Lett. 2002; 89: 186101-186104.

[23] Sharma A, MIttal J, Verma R. Langmuir 2002; 18: 10213-10220.

[24] Clarke N. Macromolecules 2005; 38: 6775-6778.

[25] Tanaka K, Yoon J S, Takahara A, Kajiyama T. Macromolecules 1995; 28: 934938.

[26] Ermi B D, Karim A, Douglas J F. J. Poly. Sci., Part. B 1998; 36: 191-200.

[27] Karim A, Slawecki T M, Kumar S K, Douglas J F, Satija S K, Han C C, Russell T P, Liu Y, Overnay R, Sokolov J, M. H. Rafailovich. Macromolecules 1998; 857-862.

[28] El-Mabrouk K, Belaiche M, Bousmina M. J. Coll. Interface Sci. 2007; 306: 354367.

[29] Nishida K, Kanaya T, Matsuba G, Ogawa H, Konishi T. Japanese Patent Pending $2005 ; 2005-058211$.

[30] El-Mabrouk K, Bousmina M. Rheol. Acta 2006; 45: 877-889.

[31] Hashimoto T, Itakura M, Hasegawa H. J. Chem. Phys. 1986; 85: 6118-6128.

[32] Reiter G. Macromolecules 1994; 27: 3046-3052.

[33] Hashimoto T, Kumaki J, Kawai H. Macromolecules 1983; 16: 641-648. 
[34] Hashimoto T, Itakura M, Shimidzu N. J. Chem. Phys. 1986; 85: 6773-6786.

[35] Chung H j, Ohno K, Fukuda T, Composto R J. Macromolecules; 2007; 40: 384388.

[36] Cahn J W, Hilliard J E. J. Chem. Phys. 1958; 28: 258-267.

[37] Cahn J W. J. Chem. Phys. 1965; 42: 93-99.

[38] Forrey C, Koberstein J, Pan D H. Interface Science 2003; 11: 211-223.

[39] Kanaya T, Ogawa H, Nishida K, Matsuba G. Book of Abstracts, Juelich Soft Matter Days, Bonn, Germany, 14-17 November, 2006. 


\section{Figure Captions}

Figure 1. Phase diagram of PS/PVME blend in bulk.

Figure 2. Time evolution of $\mathrm{OM}$ images after temperature jump to $115^{\circ} \mathrm{C}$ in two phase region from one phase region for film thickness (a): $65 \mu \mathrm{m},(\mathrm{b}): 9 \mu \mathrm{m}$, (c): $2 \mu \mathrm{m}$, (d): $466 \mathrm{~nm},(\mathrm{e}): 98 \mathrm{~nm}$ and (f): $42 \mathrm{~nm} . \quad$ Scale bars in OM images are $20 \mu \mathrm{m}$.

Figure 3. AFM images of PS/PVME blend thin films quenched to room temperature after annealing at $115^{\circ} \mathrm{C}$ in two phase region. (a): $65 \mu \mathrm{m}$ after 90 min annealing, (b): $2 \mu \mathrm{m}$ after $120 \mathrm{~min}$ annealing, (c): $466 \mathrm{~nm}$ after $90 \mathrm{~min}$ annealing and (d): $98 \mathrm{~nm}$ after 90 min annealing. Z-range is $2.0 \mu \mathrm{m}$ and $\mathrm{x}-\mathrm{y}$ size is $25 \mu \mathrm{m} \times 25 \mu \mathrm{m}$ for all images.

Figure 4. Time evolution of LS profiles after temperature jump to $115^{\circ} \mathrm{C}$ in two phase region from one phase region for film thickness (a): $65 \mu \mathrm{m},(\mathrm{b}): 9 \mu \mathrm{m},(\mathrm{c}): 2 \mu \mathrm{m}$, (d): $466 \mathrm{~nm},(\mathrm{e}): 98 \mathrm{~nm}$ and (f): $42 \mathrm{~nm}$.

Figure 5. Time evolution of (a) peak intensity $I_{\max }$ and (b) peak position $q_{\max }$ for film thickness $65 \mu \mathrm{m}, 14 \mu \mathrm{m}, 9 \mu \mathrm{m}, 2 \mu \mathrm{m}, 98 \mathrm{~nm}$ and $42 \mathrm{~nm}$.

Figure 6. Characteristic wavelength $\lambda_{\max }$ at $10 \mathrm{~min}$ (for Region I and II) and at $50 \mathrm{~min}$ (for Region IV) after temperature jump to $115^{\circ} \mathrm{C}$ in two phase region from one phase region as a function of film thickness. In Region I and II, a thick solid line shows a relation that film thickness $d$ is equal to characteristic wavelength $\lambda_{\max }\left(d=\lambda_{\max }\right)$.

Figure 7. LS profiles in the early stage of phase separation for the $65 \mu \mathrm{m}, 4 \mu \mathrm{m}$ and 2 $\mu \mathrm{m}$ films. The intensity is normalized to that at $q=5 \mu \mathrm{m}^{-1}$. 


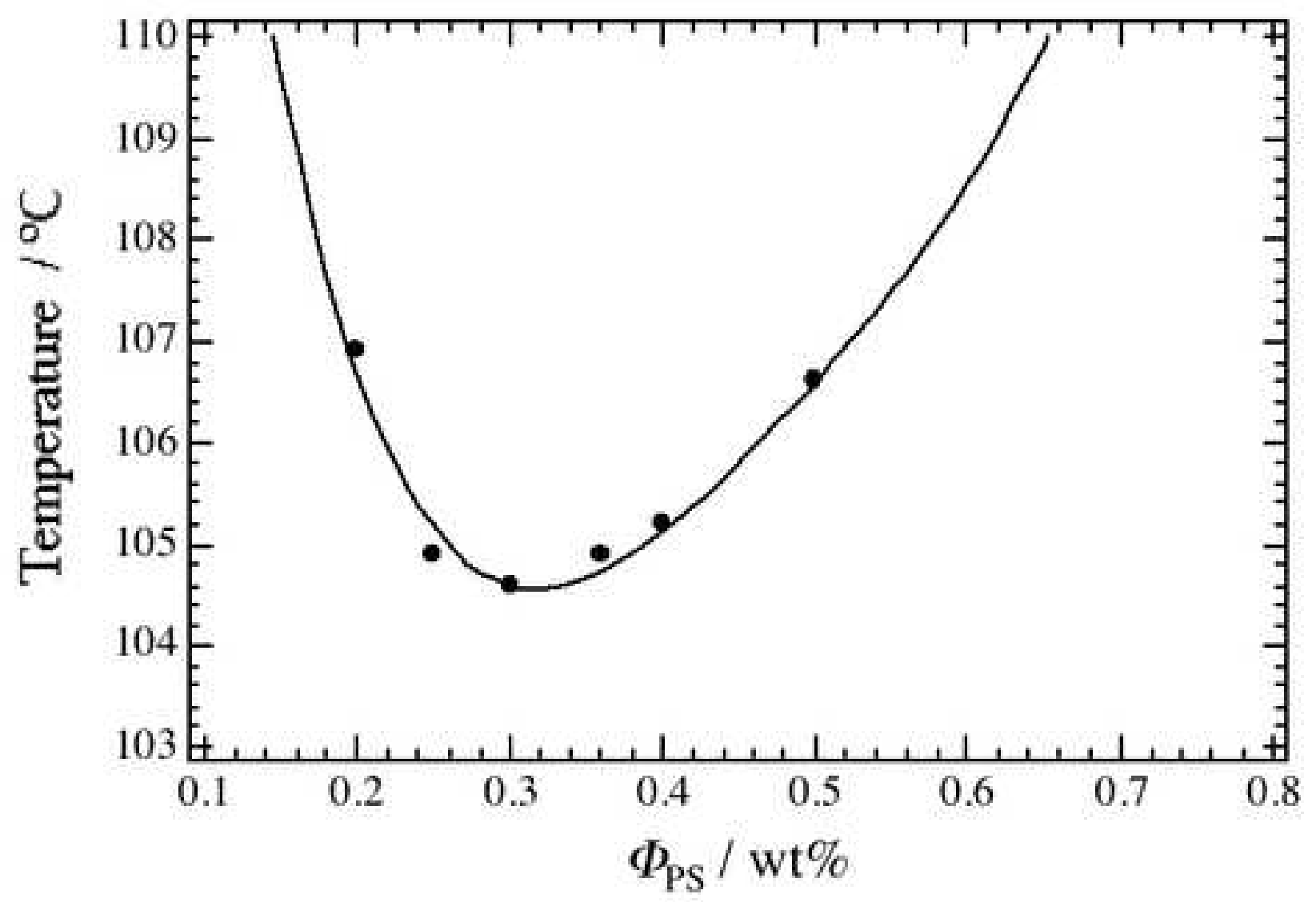




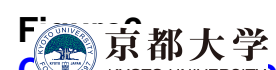

C 32 KYOTO UNIVERITYJWnload high resolution image

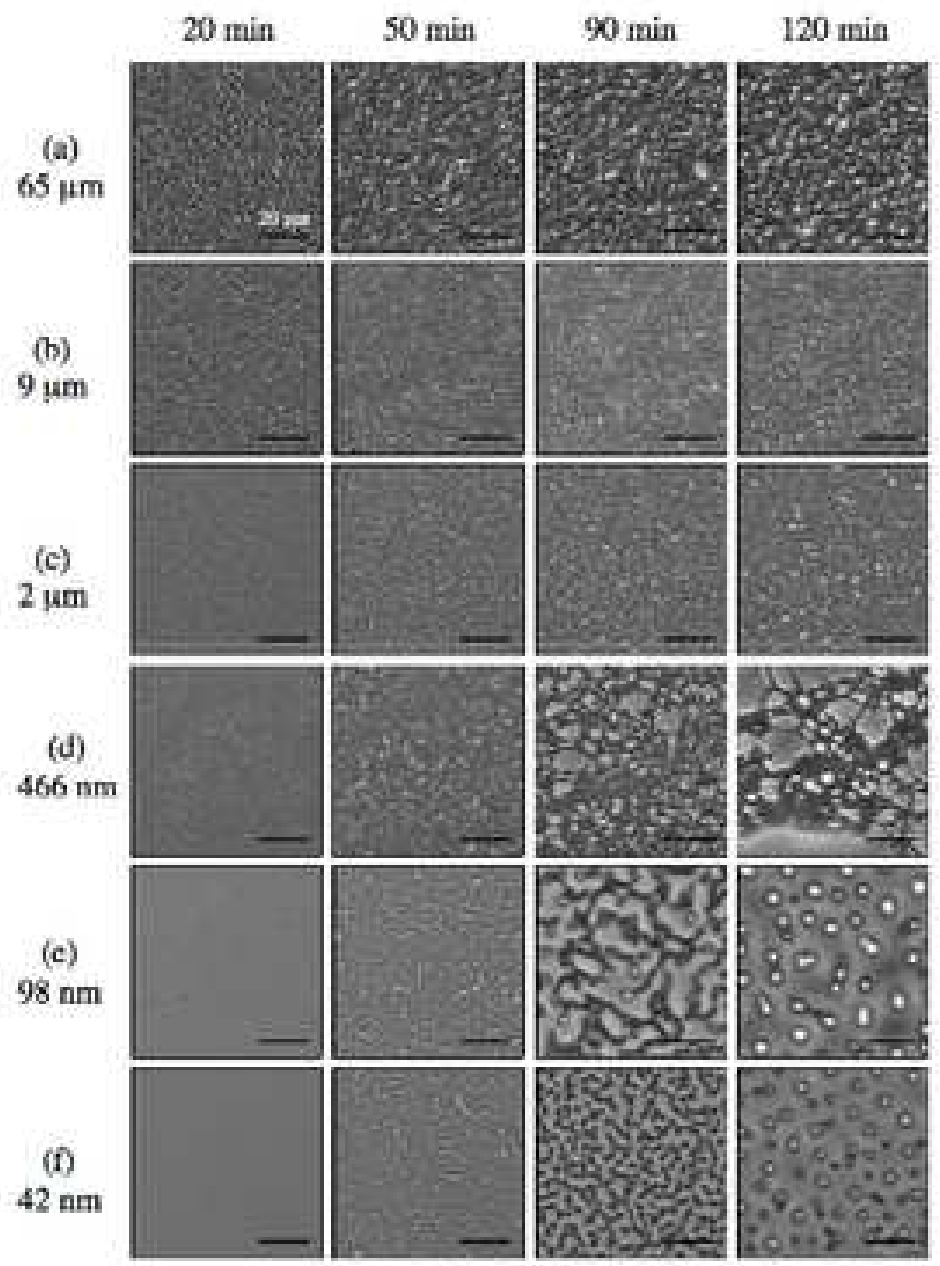




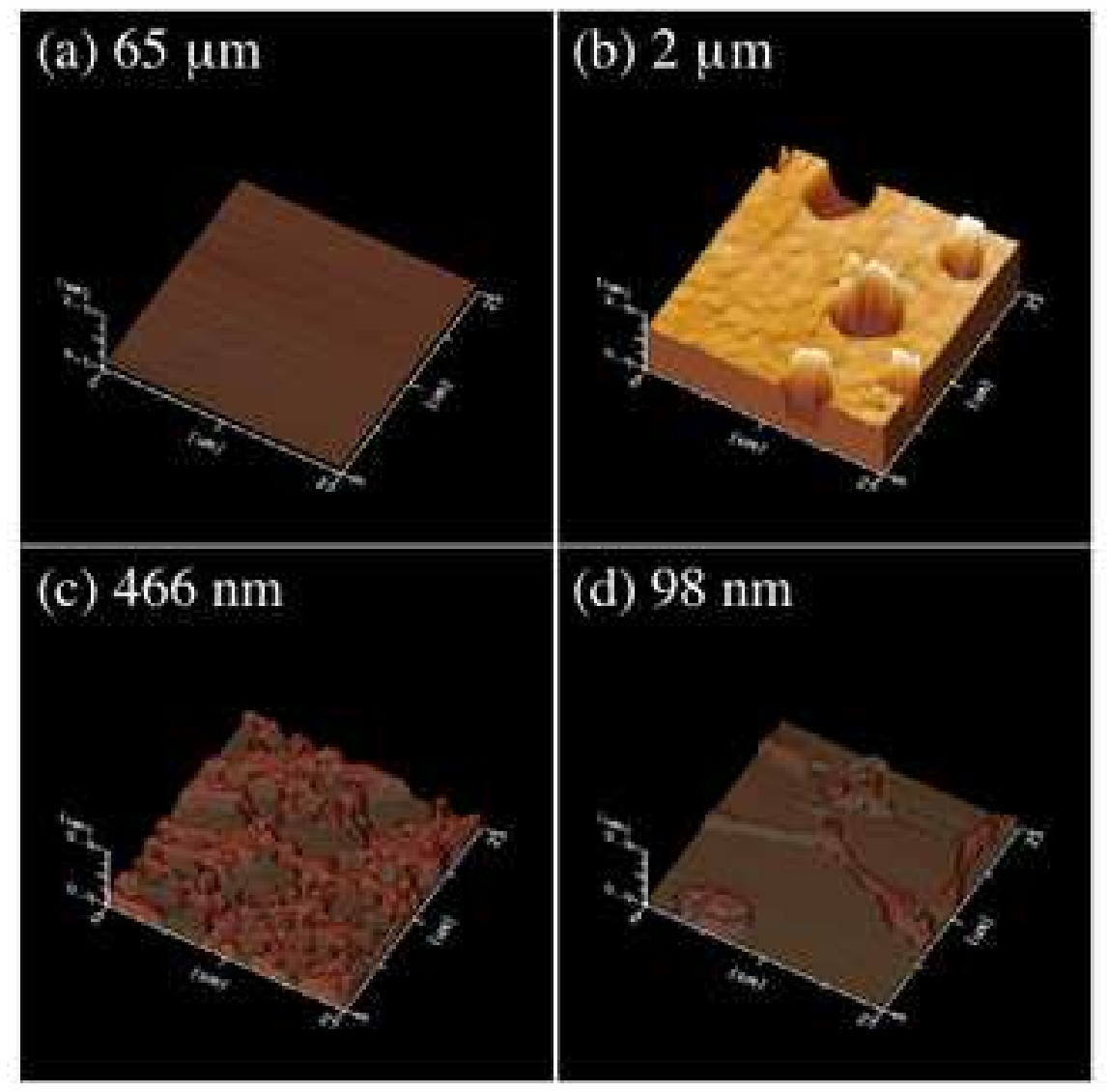

(b) $2 \mu \mathrm{m}$

(d) $98 \mathrm{~nm}$

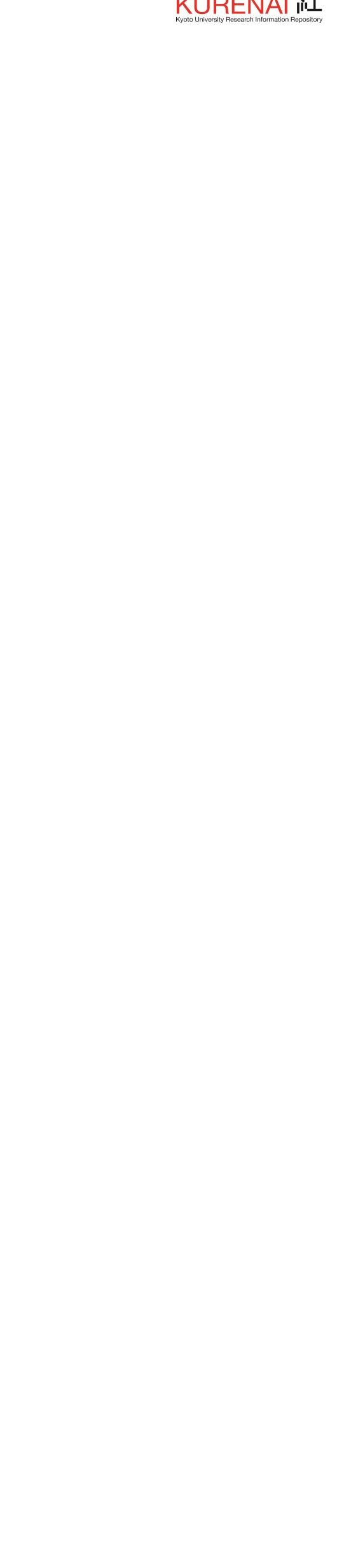


$F^{\prime}$ 新都大学

(3.) 京rorounversirtr)wnload high resolution image

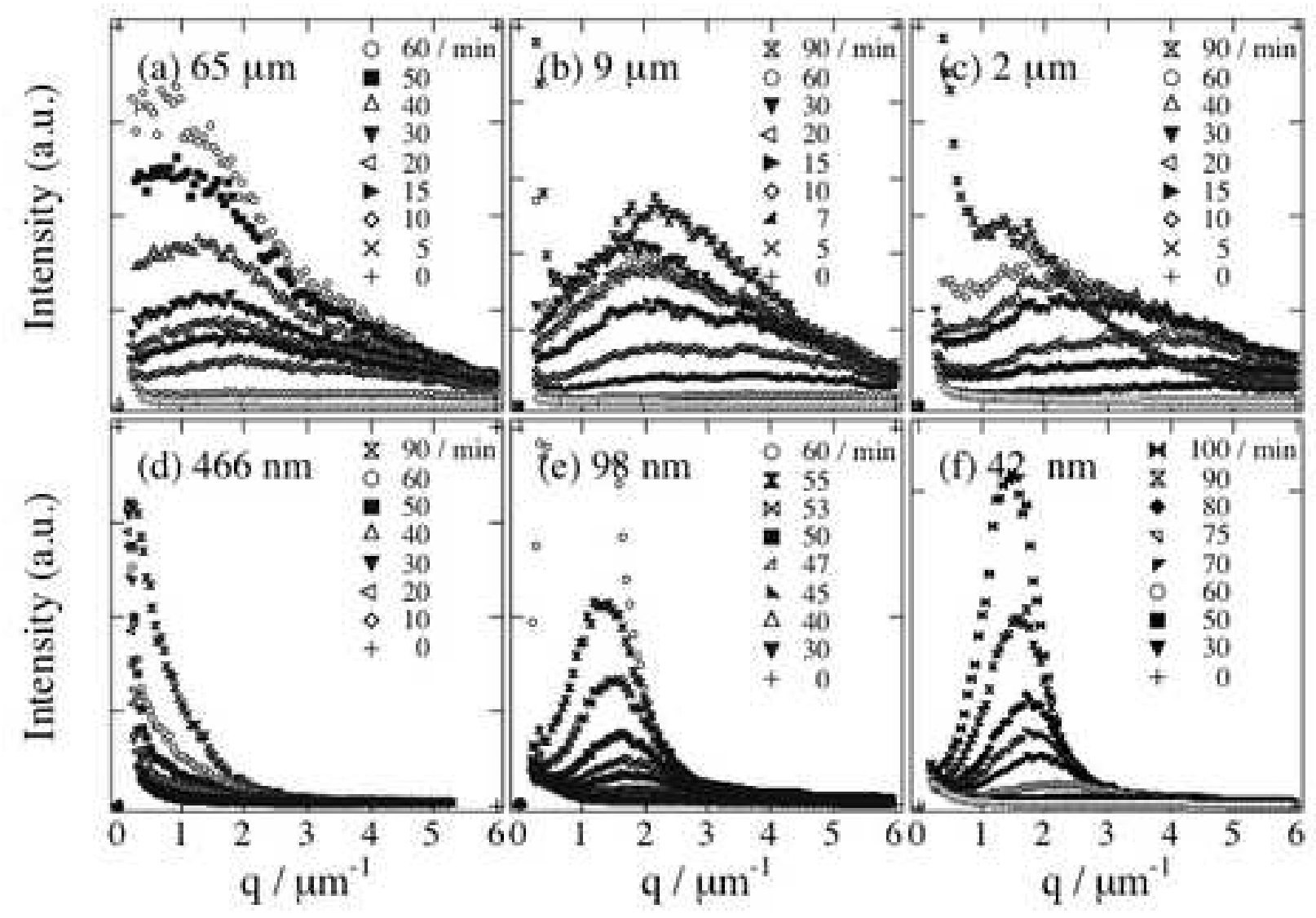

× $90 / \mathrm{min}$ (c) $2 \mu \mathrm{m} \quad \circ 60$ - $\mathbf{3}$ 30 $\approx+20$ htes.//repository.kulib.kyoto-u.ac.jp

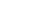

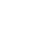

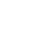

(1)

(1)

(1) 

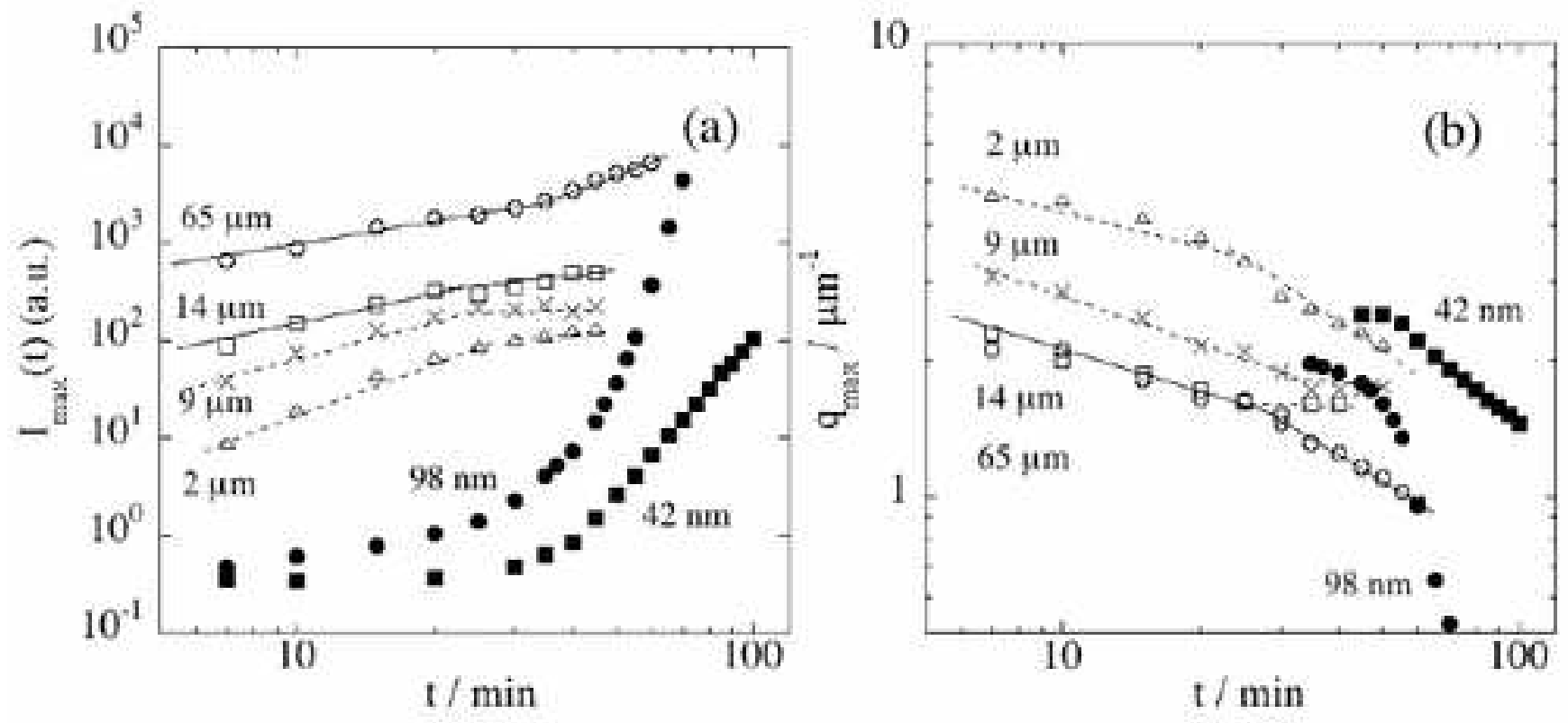


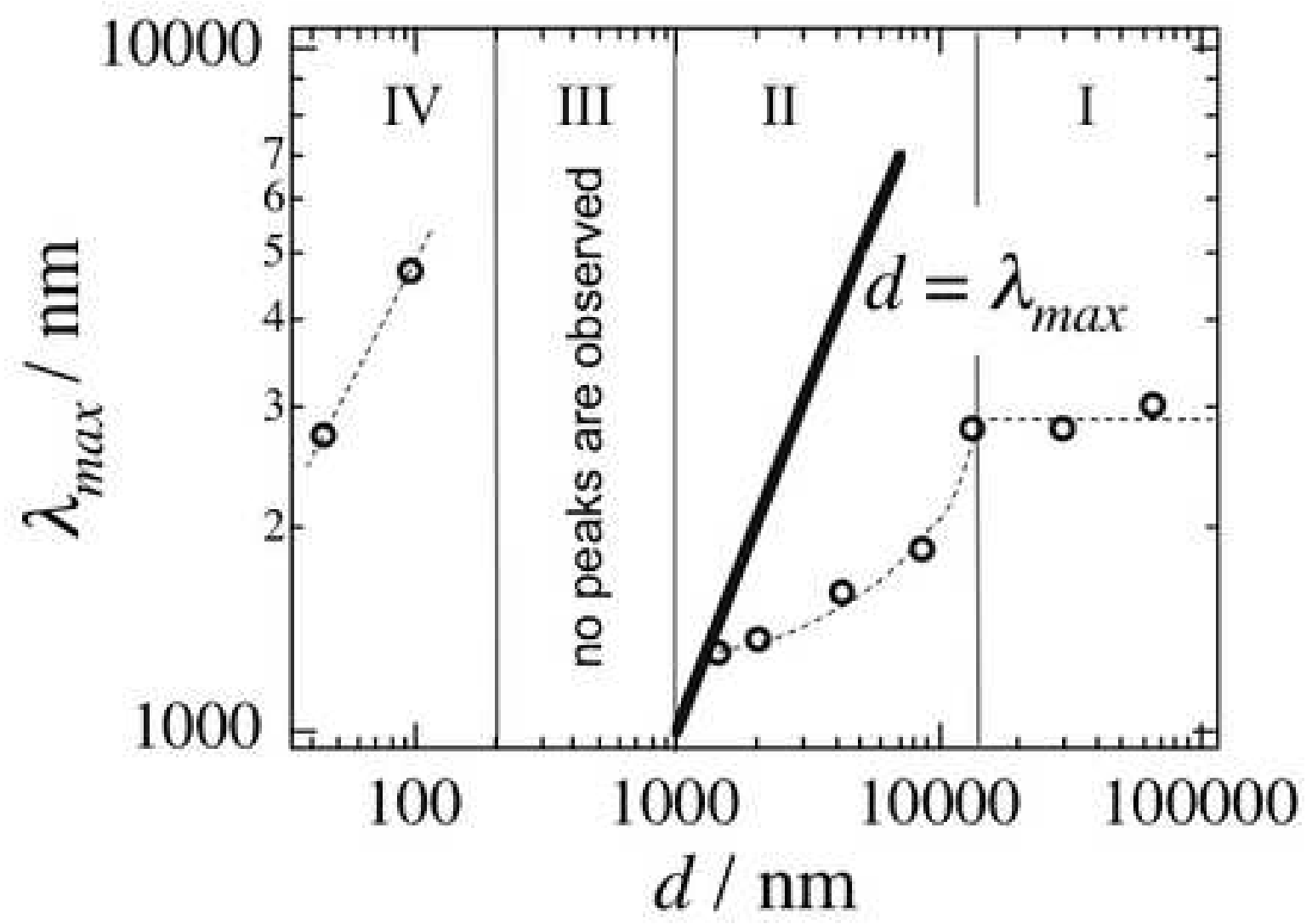

$d / \mathrm{nm}$ 


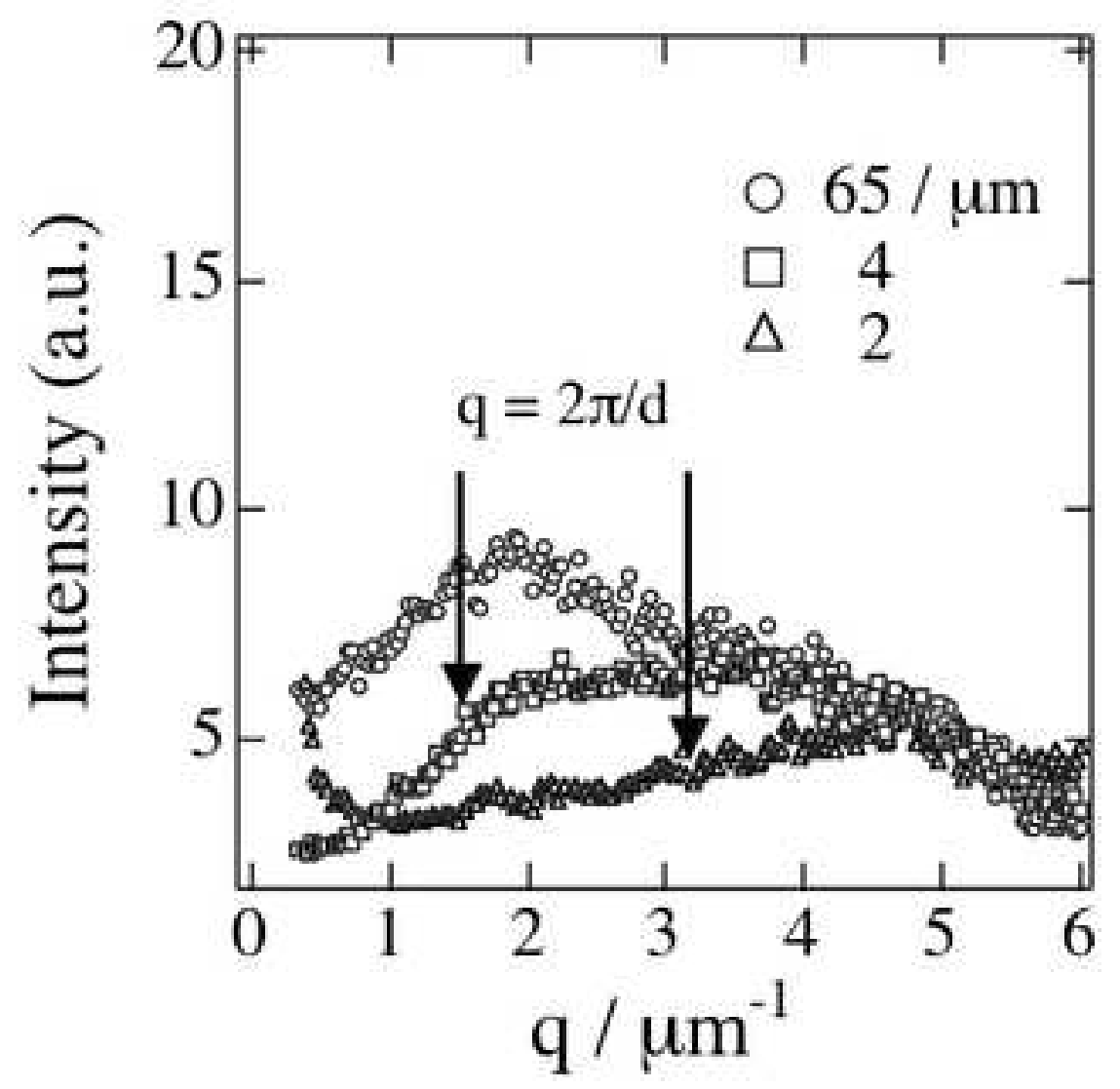

